\title{
Discrete associations of the $G C K R$ variant with metabolic risk in a Chinese population: longitudinal change analysis
}

\author{
Min Xu $\mathrm{Xu}^{1,2,3} \cdot$ Xiaofei $\mathrm{Lv}^{1,2,3} \cdot$ Lan Xie $^{4} \cdot$ Xiaolin Huang ${ }^{1,2,3} \cdot$ Ya Huang ${ }^{1,2,3}$ • \\ Ying Chen ${ }^{1,2,3}$ - Kui Peng ${ }^{1,2,3}$ - Po Wang ${ }^{1,2,3}$ - Weiqing Wang ${ }^{1,2,3}$ - Lu $\mathbf{Q i}^{5}$ • \\ Yufang $\mathrm{Bi}^{1,2,3}$ - Yimin Sun ${ }^{4,6}$. Guang Ning ${ }^{1,2,3}$
}

Received: 31 July 2015 / Accepted: 24 September 2015 /Published online: 29 October 2015

(C) Springer-Verlag Berlin Heidelberg 2015

\begin{abstract}
Aims/hypothesis Glucokinase regulatory protein gene $(G C K R)$ variant rs 780092 is a novel genetic variant associated with serum triacylglycerol (TG) identified in a genome-wide association study in East Asians. We aimed to investigate associations of rs 780092 with incident type 2 diabetes and
\end{abstract}

Min $\mathrm{Xu}$, Xiaofei Lv and Lan Xie contributed equally to this study.

Electronic supplementary material The online version of this article (doi:10.1007/s00125-015-3788-0) contains peer-reviewed but unedited supplementary material, which is available to authorised users.

Yufang Bi

byf10784@rjh.com.cn

$\checkmark$ Yimin Sun

ymsun@capitalbio.com

1 State Key Laboratory of Medical Genomics, Key Laboratory for Endocrine and Metabolic Diseases of the Ministry of Health, National Clinical Research Center for Metabolic Diseases, Collaborative Innovation Center of Systems Biomedicine, Rui-Jin Hospital, Shanghai Jiao Tong University School of Medicine, Shanghai, China

2 Shanghai Institute of Endocrine and Metabolic Diseases, Rui-Jin Hospital, Shanghai Jiao Tong University School of Medicine, Shanghai, China

3 Department of Endocrine and Metabolic Diseases, Rui-Jin Hospital, Shanghai Jiao Tong University School of Medicine, 197 Rui-Jin 2nd Road, Shanghai 200025, China

4 Department of Biomedical Engineering, Medical Systems Biology Research Center, Tsinghua University School of Medicine, 18 Life Science Park Road, Beijing 100084, China

5 Department of Nutrition, Harvard School of Public Health, Boston, MA, USA

6 National Engineering Research Center for Beijing Biochip Technology, Beijing, China dyslipidaemia, and the longitudinal changes in glucose and lipid levels.

Methods A community-based prospective cohort study was conducted at baseline in 2008, including 5,613 non-diabetic participants ( $37 \%$ male, mean age 57.6 years) with 5 years of follow-up. Blood glucose and lipid was measured at baseline and follow-up.

Results Each rs780092 T-allele was associated with a $17 \%$ lower risk of incident type 2 diabetes (HR 0.83 [95\% CI $0.73,0.95]$ ) and $36 \%$ higher risk of incident hypertriacylglycerolaemia (OR 1.36 [95\% CI 1.08, 1.72]), after adjustment for baseline fasting glucose and $\mathrm{TG}$ and other confounders. The T-allele was associated with a 5 year increasing level of $\log _{10} \mathrm{TG}(\beta \pm \mathrm{SE}, 0.01 \pm 0.004, p=0.005)$. Mediation analysis showed that both baseline TG and the 5 year increase in $\log _{10}$ TG were significant mediators in the associations of rs 780092 with risk of diabetes. The risk of incident type 2 diabetes associated with 1 SD increase in total and LDL-cholesterol was 35\% and 22\% lower in TT carriers compared with CC carriers, respectively (both $p$ for interaction $\leq 0.04)$.

Conclusions/interpretation The GCKR rs780092 variant showed opposite-directional associations with type 2 diabetes and hypertriacylglycerolaemia in a Chinese population. Both baseline level and 5 year change in serum TG were mediators of the association between the genetic variant and type 2 diabetes.

Keywords $G C K R \cdot$ Single-nucleotide polymorphism • Triacylglycerol · Type 2 diabetes
Abbreviations
F6P Fructose 6-phosphate
GCK Glucokinase 


$\begin{array}{ll}\text { GCKR } & \text { Glucokinase regulatory protein } \\ \text { GWAS } & \text { Genome-wide association study } \\ \text { HDL-c } & \text { HDL-cholesterol } \\ \text { LD } & \text { Linkage disequilibrium } \\ \text { LDL-c } & \text { LDL-cholesterol } \\ \text { SNP } & \text { Single-nucleotide polymorphism } \\ \text { TC } & \text { Total cholesterol } \\ \text { TG } & \text { Triacylglycerol }\end{array}$

\section{Introduction}

Dyslipidaemia, which is characterised by high levels of triacylglycerol (TG) and LDL-cholesterol (LDL-c), and low levels of HDL-cholesterol (HDL-c), has been related to an increased risk of type 2 diabetes. Intriguingly, several recent genome-wide association studies (GWASs) have shown that variants in glucokinase regulatory protein (GRP) gene $(G C K R)$ were related to elevated blood TG, total cholesterol (TC) and lipoprotein levels, but showed significant associations with lower glucose levels and a reduced risk of type 2 diabetes [1-3]. This discrete association with lipid and glucose metabolism has been replicated in several studies [4-9]; notably, these previous studies were performed in cross-sectional settings, mainly in white populations. Studies assessing the association of GCKR variants with incident type 2 diabetes and dyslipidaemia in prospective cohorts are sparse [8], especially those focusing on the longitudinal changes in metabolic traits, or in other ethnic groups [9].

A recent GWAS performed in East Asians for metabolic traits identified one single-nucleotide polymorphism (SNP), GCKR rs780092 (T-allele), to be associated with higher TG concentrations at the genome-wide significance level [10]. SNP rs780092 was moderately in linkage disequilibrium (LD) with the former identified variants in whites, rs780094 $\left(r^{2}=0.50\right)$ and $\mathrm{rs} 1260326\left(r^{2}=0.42\right)$ in East Asians. Whether the variant associates with type 2 diabetes, and how this variant modulates the associations between lipids and type 2 diabetes remains unclear.

In the present study, we aimed to investigate the association of the newly identified Asian-specific GCKR rs780092 variant with risk of incident type 2 diabetes and dyslipidaemia, and the longitudinal changes in glucose and lipid levels in a large Chinese cohort. Since serum TG was established as a risk factor for type 2 diabetes, we further used mediation analysis to test whether TG might be the mediator of the association between rs780092 and incident type 2 diabetes. We also examined the potential interaction of this variant with serum lipid levels on incident type 2 diabetes risk.

\section{Methods}

Study sample The present prospective and longitudinal change analysis was based on a primary cohort study designed to investigate the genetic and lifestyle/diet effects on the development of diabetes, obesity and early cardiovascular diseases. At baseline in 2008, the study participants were recruited from the Songnan community in Baoshan district, Shanghai, China. The permanent residents aged 40 and above were first invited to participate in the glucose survey. During the screening phase, the anthropometric measurements were performed and a standard questionnaire was used to collect information on lifestyle factors, history of disease and medications, etc. Fasting blood samples were collected for biochemical measurements. A total of 10,185 individuals agreed and participated in the first stage of screening. Among them, 6,919 participants provided DNA samples. The participants with DNA samples were slightly younger ( 58.0 vs 60.0 years), and more were female ( $62 \%$ vs $57 \%$ ), but they were matched in terms of BMI (both average values were $25.1 \mathrm{~kg} / \mathrm{m}^{2}$ ). For the present analysis, we excluded sequentially those with type 2 diabetes at baseline $(n=847)$ and those with missing information for rs 780092 variant $(n=66)$. In 2013, all the eligible participants $(n=6,006)$ were invited to have a follow-up examination to define their glucose metabolism status (Fig. 1). The investigation included a standard questionnaire to evaluate the changes of lifestyle factors. Anthropometric measurements and fasting blood sampling were performed. Among these eligible participants, 5,613 had blood glucose and lipid measurements taken at the follow-up examination, of which 3,509 without baseline dyslipidaemia were included for the analysis of risk of new incident dyslipidaemia and lipids change analysis (Fig. 1).

The study protocol was approved by the Institutional Review Board of Rui-Jin Hospital, Shanghai Jiao Tong University School of Medicine. All participants provided written informed consent.

Data collection and biochemical analysis Questionnaire interview and anthropometric measurements were performed at baseline and follow-up visit according to standardised protocols. Detailed information regarding lifestyles, such as tobacco smoking and alcohol drinking habits, medical history and medication use was obtained through questionnaire administered by trained physicians at baseline and 5 year follow-up examination. The participants were asked if they were a current smoker or alcohol consumer. If the answer was 'Yes', the quality and frequency of consumption were obtained. Smoking habit was defined as current ( $>6$ months on a daily basis) and non-current smoking. Alcohol intake was defined as drinker ( $>6$ months on a daily basis) or non-drinker. We grouped the participants into four groups according to their 5 year change status of smoking habits (categorical variable), 
Fig. 1 Flow chart of the study population

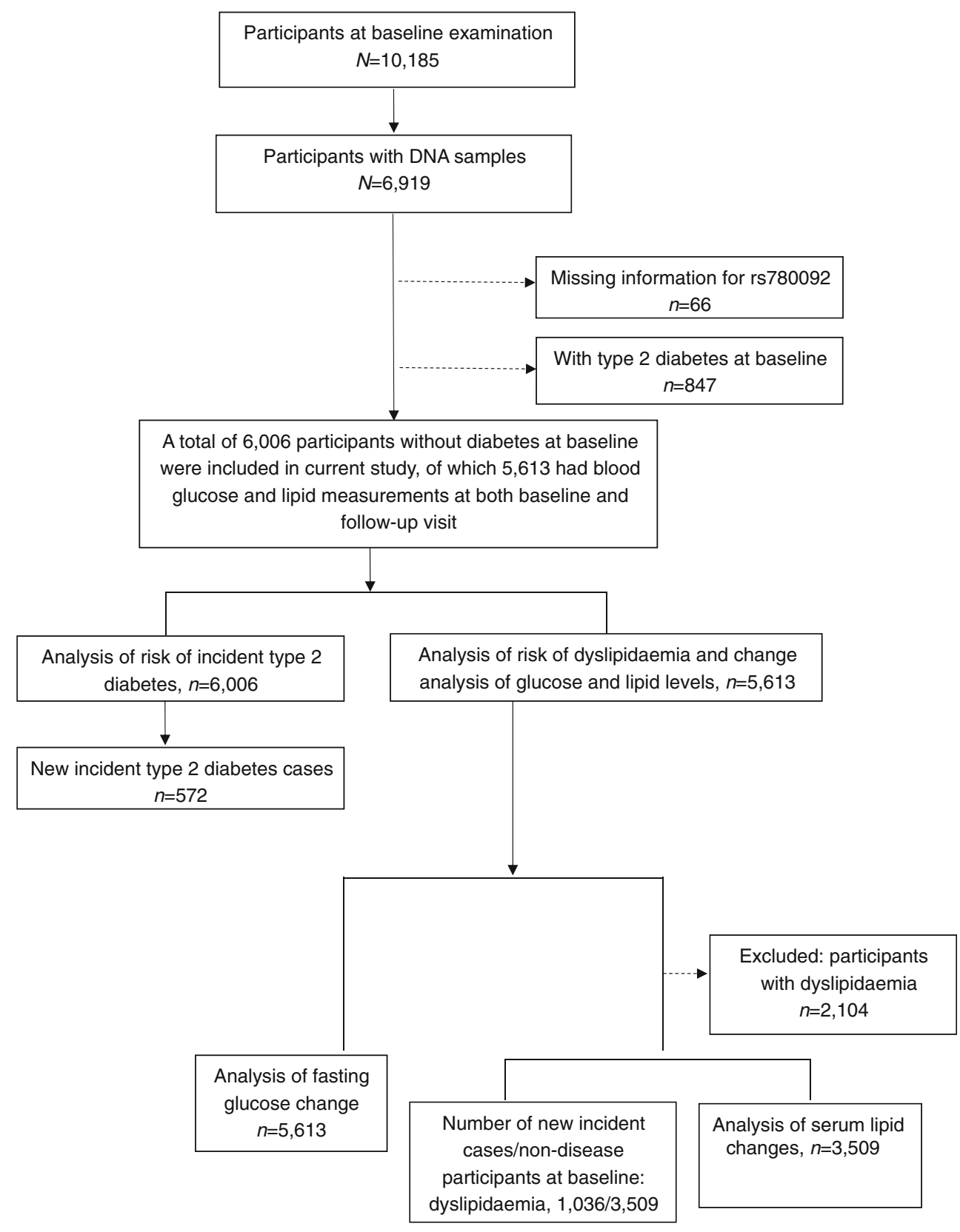

as always smoker, non-smoker, ex-smoker and new smoker. Similarly, the change of drinking habit status was categorised into always drinker, non-drinker, ex-drinker and new drinker.

Body height, weight and waist circumferences were measured by trained investigators. BMI was calculated as body weight in kilograms divided by height in metres squared. BP was measured on non-dominant arm three times consecutively with 1 min intervals after at least a 10 min rest using an automated electronic device (OMRON Model HEM-752, Omron, Dalian, China). The average of three measurements was used in the analysis.

After a minimum $10 \mathrm{~h}$ overnight fast, a venous blood sample was collected for biochemical analysis. Fasting plasma glucose concentrations were measured using the glucose oxidase method on an autoanalyser (ADVIA-1650 Chemistry System, Bayer, Leverkusen, Germany). Serum concentrations of TG, TC, HDL-c and LDL-c were also measured by an autoanalyser (ADVIA-1650 Chemistry System, Bayer).

SNP genotyping Blood white cells were collected for DNA extractions using a commercial blood genomic DNA extraction kit (OSR-M102-T1, Tiangen Biotech, Beijing, China) on an automated nucleic acid extraction instrument (OSE-M48, Tiangen Biotech) according to the manufacturer's standard protocol. Specific assays were designed using the MassARRAY Assay Design software package (v3.1) (Agena Bioscience, 
http://agenabio.com/products/massarray-system/). Mass determination was carried out with the MALDI-TOF mass spectrometer and Mass ARRAY Type 4.0 software was used for data acquisition (SEQUENOM, CapitalBio, Beijing, China). The minimum call rate was $98.7 \%$. The concordance rate was more than $99 \%$ based on genotyping 100 duplicates.

Incident cases ascertainment Ascertainment evidence of type 2 diabetes diagnosis included fasting plasma glucose and glucose-lowering medication use. The known diabetes cases at baseline were excluded. Incident type 2 diabetes was defined according to the 1999 World Health Organization (WHO) criteria (fasting plasma glucose level $\geq 7.0 \mathrm{mmol} / 1$ or receiving glucose-lowering drugs or insulin injection).

Dyslipidaemia was defined as having one of the following, according to the US National Cholesterol Education Program/ Adult Treatment Panel III (NCEP/ATP III) criteria: (1) TC $\geq 6.22 \mathrm{mmol} / 1$; (2) $\mathrm{TG} \geq 2.26 \mathrm{mmol} / \mathrm{l}$; (3) HDL-c $<1.04 \mathrm{mmol} / \mathrm{l}$; and (4) LDL-c $\geq 4.14 \mathrm{mmol} / 1$ [11]. New incident cases were defined according to the measurement of serum lipid levels at 5 year follow-up examination, after excluding baseline dyslipidaemia.

Statistical analysis Deviation from Hardy-Weinberg equilibrium for rs 780092 genotype was assessed by the $\chi^{2}$ test. Participants were categorised into three groups according to GCKR rs780092 genotypes. The comparison of differences in those baseline characteristics among three rs780092 genotypes was conducted by ANOVA for continuous variables, and $\chi^{2}$ test for categorical variables. The $p$ values were adjusted for age and sex distribution, except for the categorical variables. For the skewed distribution variables, such as serum TG, data were logarithmically transformed before statistical analysis $\left(\log _{10} \mathrm{TG}\right)$.

For incident type 2 diabetes, person-years were calculated from self-reported date of type 2 diabetes diagnosis or the respective follow-up examination date for each individual. We used the Cox time to event model to examine associations of rs 780092 with risk of type 2 diabetes. Because we had only two time-points for lipid measurements to define new dyslipidaemia cases, a logistic regression model was used to evaluate associations of the genetic variant with incident dyslipidaemia and the individual components of dyslipidaemia. Three models were used. Model 1 was unadjusted. Model 2 was adjusted for sex, baseline age, BMI, smoking and drinking status, changes in BMI, smoking and drinking habits over the follow-up period, and the baseline values for change in fasting plasma glucose, $\log _{10} \mathrm{TG}, \mathrm{TC}$, LDL-c and HDL-c, in the changes analysis. Model 3 was further adjusted for baseline fasting plasma glucose levels for the association with incident dyslipidaemia; for the association with incident type 2 diabetes, the adjustment further included baseline $\log _{10}$ TG levels.
Generalised linear models were used to examine the main effect of the genetic variant on 5 year changes in glucose and lipid traits, by fitting genotypes (additive model) as the independent variable with the corresponding lipid and glucose changes as the dependent variable.

Mediation analysis was used to test whether serum TG level (baseline or the 5 year change in $\log _{10} \mathrm{TG}$ ) might be the mediator of the association between rs780092 and incident type 2 diabetes. Figure 2 depicts the mediation analysis diagram, which shows how the effect of rs780092 on incidence of type 2 diabetes can be apportioned to the direct effect of rs 780092 on incidence of type 2 diabetes and the indirect effect via hypothesised mediators, namely $\log _{10}$ TG or the 5 year change in $\log _{10}$ TG. The risk of type 2 diabetes was modelled via the following logistic regression model:

$\operatorname{Logit}[\mathrm{P}(\mathrm{Y}=1 \mid a, m, c)]=\theta_{0}+\theta_{1 a}+\theta_{2 m}+\theta_{3 a m}+\theta^{\prime}{ }_{4 c}$

in which $a$ is exposure, $m$ is the mediator and $c$ refers to covariates.

We used the bootstrapping method to test the possibility of mediation [12]. A SAS macro was used to conduct the mediation analysis, and the $p$ values for statistical significance of mediation and the $95 \%$ bias-corrected bootstrap CI of the indirect effect were provided.

We also examined the interactions of GCKR rs780092 genotypes and baseline lipid levels on risk of incident type 2 diabetes. The significance of interactions was tested by introducing a multiplicative term of rs780092 genotype (categorical) with baseline serum lipid levels (continuous), and the lipid levels and the genotypes in the logistic regression model simultaneously, after adjustment for sex, baseline age, BMI, smoking and drinking status, and changes in BMI, smoking and drinking habits over the follow-up period. We

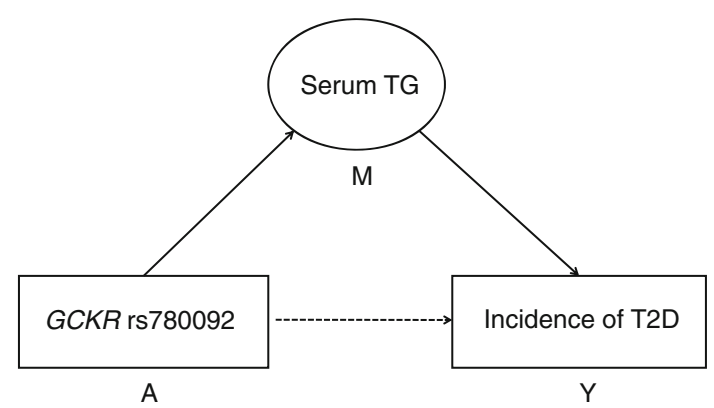

Fig. 2 Mediation analysis diagram showing how the effect of rs 780092 on the incidence of type 2 diabetes can be apportioned to the direct effect of rs780092 and the indirect effect via hypothesised mediators, namely $\log _{10}$ TG or the 5 year change in $\log _{10}$ TG. (1) The exposure variable (rs780092) affects the changes in the hypothesised mediator (serum TG) (arrow from A to M); (2) relationship between the mediator (serum TG) and the outcome (incident type 2 diabetes) (arrow from M to Y); (3) the exposure (rs780092) affects the outcome (incident type 2 diabetes) (total effect of A on Y, dotted arrow). A, exposure. M, mediator. Y, outcome. T2D, type 2 diabetes 
then conducted stratified analysis in each genotype (CC, CT and TT). Multivariable logistic regression analysis was performed to explore the association of each 1 SD increase of serum lipid levels with risk of incident type 2 diabetes in each strata of genotype.

Statistical analysis was performed using SAS version 9.3 (SAS Institute, Cary, NC, USA). All $p$ values were two-sided, and $p<0.05$ was considered statistically significant. For testing interactions, we used the Bonferroni correction to adjust for four multiple tests: $p<0.0125(0.05 / 4)$ was considered significant.

\section{Results}

Baseline characteristics of the study participants The mean age of the 6,006 participants was $57.8 \pm 9.3$ years; $37.4 \%$ were males. The major allele (T) frequency of GCKR rs780092 variant was $68.8 \%$ and the genotype distribution was in Hardy-Weinberg equilibrium $(p=0.86)$.

The baseline characteristics of participants according to GCKR genotype are shown in Table 1. The major T-allele was associated with increased TG levels at baseline ( $p$ for trend $<0.0001)$. Each T-allele was associated with 0.02 (SE, 0.005) higher $\log _{10}$ TG levels (mmol/l). Conversely, the T-allele was associated with lower fasting plasma glucose levels at baseline ( $p$ for trend=0.003). No significant differences in HDL-c, LDL-c and BMI were observed between different rs780092 genotypes.
Incidence of type 2 diabetes and the changes in metabolic traits from baseline to follow-up During the 5 year followup, 572 participants free from type 2 diabetes at baseline developed type 2 diabetes (incidence rate 0.02 ). The mean value of BMI over 5 years was increased by 0.59 (SD, 2.56) $\mathrm{kg} / \mathrm{m}^{2}$. Fasting plasma glucose, serum TG, TC and LDL-c levels were significantly increased; whereas the HDL-c levels decreased (electronic supplementary material [ESM] Table 1). Cox regression analysis showed that age at entrance, sex, baseline BMI, serum baseline TG level and drinking habits, and the 5 year increase in BMI were independently associated with risk of incident type 2 diabetes (all $p \leq 0.03$ ). Baseline TG levels were significantly associated risk of incident type 2 diabetes, with a HR, $(95 \%$ CI) of 3.47 (2.50, 4.82) $(p<0.0001)$ with each one-unit increase of $\log _{10} \mathrm{TG}$.

Discrete associations of $G C K R$ rs780092 genotype with incident type 2 diabetes and dyslipidaemia The cumulative incidence of type 2 diabetes was decreased in TT or CT genotypes, compared with that of CC genotype (Fig. 3). Cox regression analysis showed that rs 780092-T allele was significantly associated with reduced risk of diabetes. The HR $(95 \%$ CI) was $0.88(0.78,1.00)$ in the unadjusted model, and 0.83 $(0.73,0.95)$ after adjustment for sex, baseline age, BMI, current smoking and drinking, $\log _{10} \mathrm{TG}$, and changes in BMI, smoking and drinking habits over the follow-up period.

On the contrary, each T-allele was significantly associated with 36\% higher risk of incident higher TG levels (OR 1.36, $95 \%$ CI [1.08, 1.72], model 2; Table 2), after adjustment for
Table 1 Baseline characteristics of participants according to GCKR rs780092 genotypes

\begin{tabular}{lllll}
\hline Variable & CC & CT & TT & $p$ value \\
\hline$n$ & 588 & 2,573 & 2,845 & - \\
Age (years) & $57.7 \pm 9.8$ & $58.0 \pm 9.3$ & $57.7 \pm 9.2$ & 0.48 \\
Male, $n(\%)$ & $210(35.7)$ & $974(37.9)$ & $1,063(37.4)$ & 0.62 \\
BMI $\left(\mathrm{kg} / \mathrm{m}^{2}\right)$ & $25.0 \pm 4.0$ & $24.9 \pm 3.5$ & $25.0 \pm 3.9$ & 0.22 \\
Family history of diabetes, $n(\%)$ & $92(15.7)$ & $374(14.5)$ & $416(14.6)$ & 0.78 \\
Current smoker, $n(\%)$ & $143(24.3)$ & $637(24.8)$ & $690(24.3)$ & 0.91 \\
Current drinker, $n(\%)$ & $122(20.8)$ & $462(18.0)$ & $504(17.7)$ & 0.21 \\
Fasting plasma glucose (mmol/1) & $4.98 \pm 0.56$ & $4.96 \pm 0.54$ & $4.92 \pm 0.52$ & 0.01 \\
TG (mmol/l) & $1.23(0.92-1.78)$ & $1.36(0.98-1.92)$ & $1.42(1.00-2.03)$ & $<0.0001$ \\
TC (mmol/l) & $5.04 \pm 0.90$ & $5.10 \pm 0.91$ & $5.15 \pm 0.92$ & 0.006 \\
LDL-c (mmol/l) & $2.42 \pm 0.61$ & $2.46 \pm 0.66$ & $2.48 \pm 0.67$ & 0.15 \\
HDL-c (mmol/l) & $1.42 \pm 0.32$ & $1.41 \pm 0.29$ & $1.41 \pm 0.30$ & 0.91 \\
\hline
\end{tabular}

Data are unadjusted means $\pm \mathrm{SD}$, or medians (interquartile ranges) for skewed variables, or $n$ (proportions) for categorical variables

Dyslipidaemia was defined as having one of the following, according to the US National Cholesterol Education Program/Adult Treatment Panel III (NCEP/ATP III) criteria: (1) TC $\geq 6.22 \mathrm{mmol} / \mathrm{l}$; (2) TG $\geq 2.26 \mathrm{mmol} / \mathrm{l}$; (3) HDL-c $<1.04 \mathrm{mmol} / \mathrm{l}$; or (4) LDL-c $\geq 4.14 \mathrm{mmol} / \mathrm{l}$

$p$ values were calculated using ANOVA for continuous variables, and $\chi^{2}$ tests for categorical variables, after adjustment for age and sex distribution, except for the categorical variables 


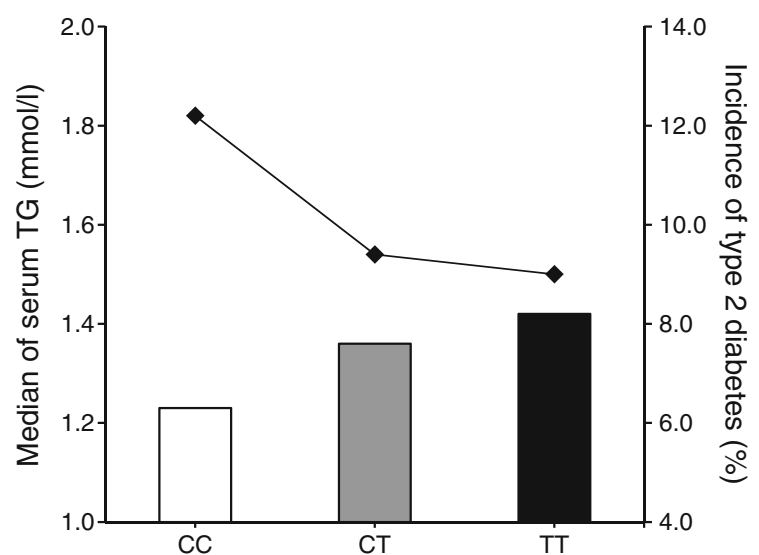

Fig. 3 Serum TG levels and incidence of type 2 diabetes according to GCKR genotypes. Bars indicate medians of TG levels at baseline. Black diamonds above bars indicate cumulative incidence of type 2 diabetes at follow-up visit

sex, baseline age, BMI, smoking and drinking status, changes in BMI, smoking and drinking habits over the follow-up period, and baseline fasting plasma glucose level (Table 2).

\section{Effects of $G C K R$ rs780092 on changes in lipid and glucose levels during follow-up}

GCKR rs780092 was also significantly associated with 5 year changes in $\log _{10}$ TG level $(\beta=0.010 \mathrm{mmol} / 1$ per T-allele, $\mathrm{SE}=$ $0.004, p=0.005$ ) after adjustment for sex, baseline age, BMI, changes in BMI, smoking and drinking habits over the followup period, and $\log _{10}$ TG levels. No significant associations were found between rs780092 and other longitudinal changes in other lipid traits or fasting plasma glucose (Table 3).

We further performed the stratified analysis, and the results were not appreciably different in participants with normal glucose tolerance and those with incident type 2 diabetes.
Mediation analysis of TG on the association between GCKR rs780092 and incident type 2 diabetes The mediation analysis showed that, after controlling for similar covariates as above, both baseline serum TG level and the 5 year changes in $\log _{10}$ TG level were significant mediators in the relationship between rs780092 and risk of type 2 diabetes (both $p<0.0001)$; the $95 \%$ CI was $(0.0012,0.0037)$ for baseline $\log _{10}$ TG and $(0.0001,0.0021)$ for 5 year changes in $\log _{10}$ $\mathrm{TG}$, respectively.

Interaction between baseline lipid levels and $G C K R$ rs780092 genotype in risk of incident type 2 diabetes We found a nominal modification effect of GCKR rs780092 genotype on association of baseline TC and LDL-c with risk of incident type 2 diabetes (both $p$ for interaction $<0.04$ ). The risk for incident type 2 diabetes associated with high baseline TC and LDL-c tended to be attenuated in carriers of T-allele as compared with $\mathrm{C}$-allele (Table 4). No obvious genetic modifications on the association of baseline TG or HDL-c with incident type 2 diabetes risk were observed (all $p$ for interaction $\geq 0.10$ ).

\section{Discussion}

In this prospective and longitudinal change analysis, we found that a GWAS-identified TG-raising GCKR rs780092 locus was significantly associated with a decreased risk of incident type 2 diabetes in a Chinese population. Mediation analysis showed both baseline TG and the 5 year increase in $\log _{10}$ TG level were significant mediators in the associations of rs780092 with type 2 diabetes. Moreover, the T-allele of rs780092 attenuated the risk of incident type 2 diabetes in relation to increased lipid levels.

Table 2 Association of GCKRrs780092 with incident type 2 diabetes and dyslipidaemia

\begin{tabular}{lcccc}
\hline Variable & Incident cases/participants & Model $1^{\mathrm{a}}$ & Model 2 $^{\mathrm{b}}$ & Model 3 $^{\mathrm{c}}$ \\
\hline Incident type 2 diabetes & $572 / 6,006$ & $0.88(0.78,1.00)$ & $0.86(0.76,0.98)$ & $0.83(0.73,0.95)$ \\
Incident dyslipidaemia & $1,036 / 3,509$ & $1.02(0.91,1.14)$ & $1.00(0.89,1.12)$ & $1.00(0.89,1.12)$ \\
$\quad$ Higher TG & $205 / 3,509$ & $1.39(1.11,1.74)$ & $1.36(1.08,1.71)$ & $1.36(1.08,1.72)$ \\
Higher TC & $454 / 3,509$ & $0.97(0.84,1.12)$ & $0.98(0.85,1.14)$ & $0.99(0.85,1.15)$ \\
$\quad$ Higher LDL-c & $267 / 3,509$ & $1.06(0.88,1.27)$ & $1.06(0.87,1.28)$ & $1.06(0.88,1.28)$ \\
Lower HDL-c & $466 / 3,509$ & $0.99(0.85,1.15)$ & $0.93(0.80,1.09)$ & $0.93(0.80,1.09)$ \\
\hline
\end{tabular}

Data are HR $(95 \% \mathrm{CI})$ for incident type 2 diabetes; and OR $(95 \% \mathrm{CI})$ for incident dyslipidaemia

${ }^{\text {a }}$ Model 1: unadjusted

${ }^{\mathrm{b}}$ Model 2: adjusted for sex, baseline age, BMI, smoking and drinking status, and changes in BMI, smoking and drinking habits over the follow-up period

${ }^{\mathrm{c}}$ Model 3: for the association with dyslipidaemia, the adjustment further included fasting plasma glucose; for the association with type 2 diabetes, the adjustment further included $\log _{10}$ TG levels

Dyslipidaemia and its components were defined according to US National Cholesterol Education Program/Adult Treatment Panel III (NCEP/ATP III) criteria: (1) $\mathrm{TC} \geq 6.22 \mathrm{mmol} / \mathrm{l}$; (2) $\mathrm{TG} \geq 2.26 \mathrm{mmol} / \mathrm{l}$; (3) HDL-c $<1.04 \mathrm{mmol} / \mathrm{l}$; or (4) LDL-c $\geq 4.14 \mathrm{mmol} / 1$ 
Table 3 Effects of GCKR rs780092 on changes in lipid and glucose levels during follow-up

\begin{tabular}{|c|c|c|c|c|c|}
\hline & Number of observations & Model $1^{\mathrm{a}}$ & $p$ value & Model $2^{\mathrm{b}}$ & $p$ value \\
\hline Change in plasma glucose & 5,613 & & & & \\
\hline$\Delta$ Fasting plasma glucose $(\mathrm{mmol} / \mathrm{l})$ & & $0.014 \pm 0.018$ & 0.43 & $0.013 \pm 0.018$ & 0.47 \\
\hline Changes in serum lipids ${ }^{c}$ & 3,509 & & & & \\
\hline$\Delta \log _{10} \mathrm{TG}(\mathrm{mmol} / \mathrm{l})$ & & $0.006 \pm 0.004$ & 0.13 & $0.010 \pm 0.004$ & 0.005 \\
\hline$\Delta \mathrm{TC}(\mathrm{mmol} / \mathrm{l})$ & & $0.003 \pm 0.02$ & 0.86 & $0.020 \pm 0.017$ & 0.24 \\
\hline$\Delta \mathrm{LDL}-\mathrm{c}(\mathrm{mmol} / \mathrm{l})$ & & $-0.0002 \pm 0.02$ & 0.99 & $0.013 \pm 0.015$ & 0.40 \\
\hline$\Delta$ HDL-c $(\mathrm{mmol} / \mathrm{l})$ & & $-0.002 \pm 0.06$ & 0.80 & $0.001 \pm 0.006$ & 0.86 \\
\hline
\end{tabular}

Data are regression coefficient $(\beta) \pm \mathrm{SE}$

${ }^{a}$ Model 1: unadjusted

${ }^{b}$ Model 2: adjusted for sex, baseline age, BMI, changes in BMI, smoking and drinking habits over the follow-up period, and the baseline values for changes in fasting plasma glucose, $\log _{10} \mathrm{TG}, \mathrm{TC}, \mathrm{LDL}-\mathrm{c}$ and HDL-c

${ }^{\mathrm{c}}$ Analysis was performed in participants without known diabetes or dyslipidaemia, and with two measurements of those metabolic traits available $p$ values were calculated from generalised linear regression models, with GCKR genotype as the independent variable and the corresponding lipid and glucose changes as dependent variables

Several studies have reported a reciprocal association of $G C K R$ variants with serum TG levels and risk of type 2 diabetes, including GWASs and subsequent replication studies. Most of these studies examined the two index-SNPs, rs780094 and rs1260326, which were identified in whites and are moderately in LD with rs780092, as investigated in the present study. The present study was the first to investigate the associations of rs780092 with risk of incident dyslipidaemia and type 2 diabetes. Our findings are in line with results from previous studies in whites and Asians, in which the $G C K R$ variant reversely associated with TG levels and type 2 diabetes risk $[4,6,9,13]$. Notably, one prospective cohort study has shown that the T-allele of rs1260326 was strongly associated with lower fasting glucose and, conversely, higher TG during a 9 year study [9]. Another prospective study also demonstrated that the rs1260326 variant was associated with longitudinal changes in TG but not fasting plasma glucose levels [4]. Similarly, a significant association between rs780092 and 5 year change in $\log _{10}$ TG was also observed in our study, although rs780092 was not significantly associated with increasing levels of fasting plasma glucose.

Our mediation analysis indicated that the high TG pathway might partially mediate the effect of the GCKR rs780092 variant on type 2 diabetes, for which the mechanisms remain unestablished. First, physiologically, the GCKR gene product GCK regulatory protein (GKRP), is predominantly expressed in the liver and inhibits the activity of GCK. With increased GCK activity, hepatic glucose utilisation is increased and fatty acid synthesis is upregulated [14]. These changes in turn promote de novo lipogenesis and VLDL-TG production and
Table 4 Association of each 1SD increase in baseline lipid levels with risk of incident type 2 diabetes stratified by GCKR genotypes

\begin{tabular}{lllll}
\hline Variable & $\mathrm{CC}$ & $\mathrm{CT}$ & $\mathrm{TT}$ & $p$ for interaction \\
\hline Incident cases $(\%)$ & $72(12.2 \%)$ & $243(9.4 \%)$ & $257(9.0 \%)$ & \\
TG $(\mathrm{mmol} / \mathrm{l})$ & $1.55(1.24,2.01)$ & $1.17(1.04,1.30)$ & $1.24(1.13,1.37)$ & 0.10 \\
$\quad p$ value & 0.0005 & 0.006 & $<0.0001$ & \\
TC $(\mathrm{mmol} / \mathrm{l})$ & $1.52(1.19,1.95)$ & $1.29(1.13,1.47)$ & $1.17(1.03,1.33)$ & 0.03 \\
$\quad p$ value & 0.0008 & 0.0002 & 0.02 & \\
LDL-c $(\mathrm{mmol} / \mathrm{l})$ & $1.35(1.05,1.74)$ & $1.33(1.16,1.51)$ & $1.13(0.99,1.28)$ & 0.04 \\
$\quad p$ value & 0.02 & $<0.0001$ & 0.07 & \\
HDL-c $(\mathrm{mmol} / \mathrm{l})$ & $0.96(0.71,1.28)$ & $0.87(0.74,1.02)$ & $0.87(0.75,1.01)$ & 0.48 \\
$\quad p$ value & 0.79 & 0.08 & 0.06 & \\
\hline
\end{tabular}

Data are ORs $(95 \% \mathrm{CI})$

Data were calculated using logistic regression model after adjustment for sex, baseline age, BMI, smoking and drinking status, and changes in BMI, smoking and drinking habits over the follow-up period

$p$ values were tested for linear trend of each 1 SD increase of lipid levels with risk of type 2 diabetes in each genotype category

$p$ for interaction was derived from logistic regression analysis, by introducing a multiplicative term of continuous lipid levels and GCKR genotype, the lipid levels and the genotypes in the model simultaneously 
suppress hepatic glucose output [15]. Second, and more importantly, the GCKR rs780092 might have a direct effect on glucose metabolism. GKRP also regulates concentrations of fructose 6-phosphate (F6P), which can enhance GCK-CKRP complex formation $[14,16]$. The GCKR variant (rs120326 [c.1337C > T; p.P446L]) produces GKRP that has reduced nuclear sequestration and F6P-mediated inhibition of GCK, which leads to increased GCK activity, resulting in increased hepatic glucose disposal and the subsequent decreased plasma glucose concentrations [17]. Such a biological mechanism might provoke new strategies in glucose-lowering drug design. Recently, two GKRP disruptors have already demonstrated great potential for glucose-lowering therapy [18].

The major strengths of our study include the prospective study design, the 5 year changes analysis in blood glucose and lipids, and a large well-defined, community-based cohort. Moreover, it is the first report of a TG-associated genetic variant identified in East Asians being associated with a lower risk of incident type 2 diabetes. The association was partially mediated by serum TG levels. There are several limitations in our study that should be acknowledged. First, the definition of type 2 diabetes was based on fasting glucose testing. Absence of an OGTT might have misclassified participants with type 2 diabetes into the non-diabetic group at baseline and underestimated new cases during follow-up. Second, our population is limited to middle aged and elderly Chinese participants; therefore, it should be cautiously generalised to other demographic groups or ethnicities.

In conclusion, we found that the TG-raising GCKR rs780092 T-allele was significantly associated with an increased risk of incident hypertriacylglycerolaemia and a decreased risk of incident type 2 diabetes in a prospective cohort of Chinese. The gene-diabetes association was partially mediated by serum TG levels. The T-allele of the variant might attenuate the incident type 2 diabetes risk associated with increased lipid levels. The associations of GCKR rs780092 genotype with dyslipidaemia and incident type 2 diabetes might infer potential mechanisms underlying the pathogenesis for type 2 diabetes and new strategies in glucose-lowering therapy.

Acknowledgements The authors would like to acknowledge all participants for their cooperation.

Funding This work was supported by grants from the China National Clinical Research Center for Metabolic Diseases [2013BAI09B13], 973 Program of China [2015CB553600], National Key New Drug Creation and Manufacturing Program of Ministry of Science and Technology of China [2012ZX09303006-001], the National High Technology Research and Development Program of China (863 Program) [2012AA02A509 and 2012AA020101] and the National Natural Science Foundation of China [81471062, 81321001, 81390350, 81222008 and 81270877], the Joint Research Program for Important Diseases of the Shanghai Municipal Commission of Health and Family Planning (2013ZYJB1002) and the Shanghai Pujiang Project (14PJD024).
Duality of interest The authors declare that there is no duality of interest associated with this manuscript.

Author contribution MX, XL, LQ and GN contributed to the study concept and design. Acquisition of data was performed by XL, LX, XH, $\mathrm{YH}, \mathrm{YC}, \mathrm{KP}, \mathrm{PW}$ and $\mathrm{YS}$. The analysis and interpretation of data was carried out by MX, XL, WW, YB and LQ. MX, XL and LX drafted the manuscript; XH, YH, YC, KP, PW, WW, YB, YS and GN critically revised the manuscript for important intellectual content. All authors gave final approval of the version to be published. GN is responsible for the integrity of the work as a whole.

\section{References}

1. Diabetes Genetics Initiative of Broad Institute of Harvard and MIT, Lund University, and Novartis Institutes of BioMedical Research, Saxena R, Voight BF et al (2007) Genome-wide association analysis identifies loci for type 2 diabetes and triglyceride levels. Science 316:1331-1336

2. Teslovich TM, Musunuru K, Smith AV et al (2010) Biological, clinical and population relevance of 95 loci for blood lipids. Nature 466:707-713

3. Dupuis J, Langenberg C, Prokopenko I et al (2010) New genetic loci implicated in fasting glucose homeostasis and their impact on type 2 diabetes risk. Nat Genet 42:105-116

4. Orho-Melander M, Melander O, Guiducci C et al (2008) Common missense variant in the glucokinase regulatory protein gene is associated with increased plasma triglyceride and C-reactive protein but lower fasting glucose concentrations. Diabetes 57:3112-3121

5. Stančáková A, Paananen J, Soininen P et al (2011) Effects of 34 risk loci for type 2 diabetes or hyperglycemia on lipoprotein subclasses and their composition in 6,580 nondiabetic Finnish men. Diabetes 60:1608-1616

6. Sparso T, Andersen G, Nielsen T et al (2008) The GCKR rs780094 polymorphism is associated with elevated fasting serum triacylglycerol, reduced fasting and OGTT-related insulinaemia, and reduced risk of type 2 diabetes. Diabetologia 51:70-75

7. Li N, van der Sijde MR, Bakker SJ et al (2014) Pleiotropic effects of lipid genes on plasma glucose, HbA1c, and HOMA-IR levels. Diabetes 63:3149-3158

8. Vaxillaire M, Cavalcanti-Proença C, Dechaume A et al (2008) The common P446L polymorphism in GCKR inversely modulates fasting glucose and triglyceride levels and reduces type 2 diabetes risk in the DESIR prospective general French population. Diabetes 57:2253-2257

9. Tam CH, Ma RC, So WY et al (2009) Interaction effect of genetic polymorphisms in glucokinase (GCK) and glucokinase regulatory protein (GCKR) on metabolic traits in healthy Chinese adults and adolescents. Diabetes 58:765-769

10. Kim YJ, Go MJ, Hu C et al (2011) Large-scale genome-wide association studies in East Asians identify new genetic loci influencing metabolic traits. Nat Genet 43:990-995

11. Expert Panel on Detection, Evaluation, and Treatment of High Blood Cholesterol in Adults (2001) Executive Summary of the Third Report of the National Cholesterol Education Program (NCEP) Expert Panel on Detection, Evaluation, and Treatment of High Blood Cholesterol in Adults (Adult Treatment Panel III). JAMA 285:2486-2497

12. Preacher KJ, Hayes AF (2008) Asymptotic and resampling strategies for assessing and comparing indirect effects in multiple mediator models. Behav Res Methods 40:879-891

13. Qi Q, Wu Y, Li H et al (2009) Association of GCKR rs780094, alone or in combination with GCK rs1799884, with type 2 diabetes and related traits in a Han Chinese population. Diabetologia 52:834-843 
14. Beer NL, Tribble ND, McCulloch LJ et al (2009) The P446L variant in GCKR associated with fasting plasma glucose and triglyceride levels exerts its effect through increased glucokinase activity in liver. Hum Mol Genet 18:4081-4088

15. Gerich JE (1993) Control of glycaemia. Baillieres Clin Endocrinol Metab 7:551-586

16. Rees MG, Wincovitch S, Schultz J et al (2012) Cellular characterisation of the GCKR P446L variant associated with type 2 diabetes risk. Diabetologia 55:114-122
17. Raimondo A, Rees MG, Gloyn AL (2015) Glucokinase regulatory protein: complexity at the crossroads of triglyceride and glucose metabolism. Curr Opin Lipidol 26:88-95

18. Lloyd DJ, St Jean Jr DJ, Kurzeja RJM (2013) Antidiabetic effects of glucokinase regulatory protein small-molecule disruptors. Nature 504:437-440 